\title{
Tackling the shadow pandemic
}

\author{
As COVID-19 continues to surge, it is essential to understand and address the looming crisis of mental-health \\ issues caused or exacerbated by the pandemic.
}

$\Lambda$

lmost a year since the first COVID-

19 case was reported, the dark

undercurrent of the pandemic is its immeasurable toll on the mental health and well-being of people around the globe.

Past viral outbreaks have been linked to adverse effects on mental health. For example, depression, insomnia and anxiety were common symptoms among people with confirmed infection during the 2003 SARS and 2012 MERS outbreaks both in the acute-illness phase and in the post-illness phase, with post-traumatic stress disorder affecting about a third of people who recovered ${ }^{1}$. People who have contracted COVID-19, those who face increased risk of severe illness because of their age or underlying medical conditions, and people who already experience mental ill-health are more vulnerable to mental-health issues related to the pandemic. COVID-19 itself can have neurological and mental-health effects, including agitation, delirium and stroke, but the scale and protracted nature of the current outbreak, together with the wide-ranging presentation and severity of COVID-19 among different patients, increases the number and complexity of stressors, which raises the possibility of mental-health problems for a larger part of the population. According to a KFF Tracking Poll conducted in the USA in the summer, $53 \%$ of adults surveyed said that their mental health was negatively impacted by worry and stress over the coronavirus, with roughly a third reporting issues with sleep and eating habits and $12 \%$ noting an increase in alcohol or substance use. A separate US study conducted around the same time reported that $\sim 11 \%$ of respondents had seriously considered suicide in the 30 days prior to the survey ${ }^{2}$. Both reports noted that the pandemic also affects different population groups disproportionately when it comes to mental health, with healthcare workers, women, young adults and racial/ethnic groups, including Black and Hispanic people, being more likely to be negatively impacted by COVID-19-related mental-health issues.

Healthcare workers in particular were found to be at increased risk of trauma, stress-related disorders, anxiety and depression during the SARS and MERS outbreaks, and also during the current pandemic ${ }^{3}$. The emotional toll of caring for large numbers of patients with limited treatment options, shortages in medical personnel and protective equipment, and fear of exposing oneself and loved ones to the virus is enormous.

Furthermore, outbreak-containment strategies, such as imposing quarantine on people who might have been exposed to infection, are known to have negative psychological effects, including stress, depression, insomnia and symptoms of post-traumatic stress ${ }^{4}$. The lack of effective therapies and the high contagiousness of SARS-CoV-2 led to the wide adoption of quarantine and lockdown public-health measures throughout the year to stem the spread of the virus. However, the prolonged nature of some home-confinement directives and the resulting isolation and loneliness have placed people at greater risk of mental-health problems. The potential impact of social isolation and school closures on the mental health of children and teenagers may be hard to estimate in the short term but is also a major cause for concern.

The knock-on effect of the pandemic on the global economy and the job insecurity, loss of income and financial struggles this has caused are also major stressors for a large part of the population. In the July KFF tracking poll, $71 \%$ of responders who had difficulty paying household bills due to the financial impact of COVID-19 reported negative effects on their mental health due to worry and stress. Conversely, having to juggle work with increased caregiving responsibilities - whether for homeschooling or caring for sick family members - can be a major additional source of stress for others. The botched response to COVID-19 in many countries and mixed or conflicting messages from government and public-health officials, including the politicization of simple public-health measures such as the wearing of face coverings, have also created social tensions and heightened anxiety. Finally, the grief for the lives lost and the devastating death toll of this disease cannot be overstated.

As governments and health organizations have focused - with variable success - on pressing needs directly related to COVID19 , such as controlling the spread, treating patients, and developing and testing therapies and vaccines, mental-health needs and services have suffered. Although a recent report by the World Health Organization on the impact of COVID-19 on mental, neurological, and substance-use (MNS) services in 130 countries noted that $89 \%$ of responding countries included mental health and psychosocial support in their COVID-19 response planning, only $17 \%$ allocated additional funding for such efforts. Mental health is generally underfunded, with countries earmarking only $\sim 2 \%$ of their health budgets for mental health. Thus, it is not surprising that the stress test imposed on health systems by COVID-19 resulted in $93 \%$ of surveyed countries reporting disruptions in MNS services, with roughly a third also noting some disruption in emergency care and supply of medications for people with MNS disorders.

Despite the recent positive news about vaccine development, the harsh reality is that we are not yet close to the long-awaited 'return to normal', especially as Europe and the Americas are fighting to control a second wave of infections. Thus, it is essential to intensify efforts to understand and mitigate the unfolding mental-health crisis. Priorities should include increasing investment in mental-health systems and professionals; using technology to identify those most at risk of adverse mental-health outcomes and offer them appropriate resources and long-term support; and continuing the collection and analysis of data to understand the complex relationship among COVID-19, mental health and existing health systems. At the level of the individual person, practicing self-care, being mindful of one's own mental state and stressors and developing coping strategies and support networks of people and resources is important. In March of this year, the World Health Organization published a set of considerations to support the mental well-being of people in various groups of the population during the COVID-19 outbreak. Translating these approaches into our interactions with others will build stronger social networks and help those who may be more vulnerable to adverse mental-health effects.

\footnotetext{
Published online: 16 December 2020 https://doi.org/10.1038/s43018-020-00162-9

References

1. Rogers, J. P. et al. Lancet Psychiatry 7, 611-627 (2020).

2. Czeisler, M. É. et al. MMWR Morb. Mortal. Wkly. Rep. 6, 1049-1057 (2020).

3. Cabarkapa, S., Nadjidai, S. E., Murgier, J. \& Ng, C. H.

Brain, Behavior, \& Immunity - Health 8, 100144 (2020).

4. Brooks, S. K. et al. Lancet 395, 912-920 (2020).
} 